blissements hospitaliers contenant $7605 \mathrm{lits,}$ un nombre indéterminé d'hôpitaux dans la banlieue de Bordeaux et un ensemble d'hôpitaux répartis dans tout le département, qui sera subdivisé, à cet effet, en six sections. Le premier groupe des hòpitaux de la ville de Bordeaux, qui est prêt à fonctionner, comprend : une infirmerie de gare, un hôpital de répartition el 21 hòpitaux auxiliaires. Ce vaste système d'hospitalisation sera étendu, autant que possible, dans tout le département, de manière que la répartition des blessés se fasse d'une manière régulière et qu'ils soient tous assurés de recevoir les soins que leur état réclame.

Le Conseil central de la Société française étudie la constitution régionale de plusieurs ambulances auxiliaires de campagne; le Comité de la Gironde a réclamé et obtenu l'honncur de former l'une de ces ambulances.

\title{
GRANDE-BRETAGNE
}

\section{DU PREMIER PANSEMENT PAR LE BLESSÉ LUL-MÈME}

Notre digne et savant collègue Sir Thomas Longmore, professeur de chirurgie à l'école militaire de Netley, vient de traiter, dans un court mémoire ${ }^{1}$, des assortiments d'objets de premier pansement à porter par le soldat en campagne.

L'habitude de faire porter, par chaque soldat en service actif, ce qu'il faut pour un premier pansement, remonte, pour l'armée britannique, à l'année 1855, époque de la guerro de Crimée. Depuis lors il en a été de même tontes les fois que les troupes anglaises sont entrées en campagne; cet usage s'est aussi introduit plus ou moins généralement dans les armées du continent. Des objets pour le premier pansement font partie, par exemple, de l'équipement réglementaire de l'armée allemande. Des chirurgiens éminents ont également, en différentes occasions, discuté à fond la question de savoir de quoi doit se composel ce matériel sanitaire.

1 Voir aux Ouvrages recus. 
Mais, dans ces dernières années, des doutes sérieux ont été émis sur l'opportunité de la mesure elle-même, entr'autres par le médecin général $\mathrm{D}^{\mathrm{r}}$ Roth de Dresde, par le Dr Gori d'Amsterdam, par le $D^{r}$ Roches, chirurgien d'état major en Allemagne; enfin, le médecin-major $D^{r}$ E. Delorme, de l'armée française, a dit explicitement que celte adjonction constitue, pour le soldat, une surcharge inutile, et, pour l'Etat, une perte sèche. Mais il faut ajouter que d'autres chirurgiens militaires également éminents se sont prónoncés pour l'affirmative.

C'est pour contribuer à résoudre définitivement cette importante question que le professeur Longmore a publié le mémoire dont nous nous faisons un devoir de donner ici un résumé, malgré son caractère technique, pensant que la plupart de nos lecteurs seront bien aise de connaître l'opinion de l'auteur sur un problème qui touche de près à l'œuvre de la Croix-Rouge.

Il s'agit d'élucider trois points :

$1{ }^{\circ} L^{\prime}$ expérience a-t-elle démontré la nécessité, pour le soldatblessé, des objets de premier pansement qu'il porte sur lui?

$2^{\circ}$ Les doutes qu'on a élevés sur la convenance de faire porter ce pelit assortiment au soldat lui-même sont-ils fondés?

$3^{0}$ Si la mesure est bonne en elle-même, y a-t-il quelque chose à faire pour atténuer ou supprimer certains inconvénients qui ont été signalés?

Le lecteur comprendra que nons ne puissions reproduire ici tous les détails de l'étude à laquelle s'est livré le professeur Longmore, quelque concise et substantielle qu'elle soit.

La nécessité d'un premier pansement provisoire n'est pas douteuse. Il est évident qu'une blessure qui n'est pas compliquée d'hémorrhagie doit ètre préservée des causes extérieures d'infection, et, autant que possible, protégée contre de nouvelles violences. Cela répond d'ailleurs à l'instinct du blessé lui-même. L'ordonnance du ministère de la guerre anglais prescrit formellement que le soldat sera outillé lui-même pour cela. En effet, l'expérience des dernières guerres a montré que, si le sldal ne les porte pas sur lui, ces objets de première nécessité, quelqu'abondants qu'ils soient, ne se trouvent pas a sa portée, an moment et à l'endroit où il en a un besoin immédiat.

Ces approvisionnements chirurgicaux faits par l'intendance ne laissent rien à désirer, mais tout le monde doit reconnaître que, 
très souvent, ils se trouvent dans des dépôts éloignès de la place où un combat inattendu les rend le plus nécessaires. Ce grave inconvénient, que l'auteur a observé lui-même daus la guerre de Crimée, se reproduirait inévitablement si l'on ne chargeait le soldat de transporter les objets peu voluminenx, mais indispensables, dont il s'agit.

Il est vrai que, par suite des progrès modernes de la stratégie médicale, les places de pansement sont plus largement approvisiomnées qu'auparavant, mais cet avantage, qui est réel, est compensé par l'immense extension qu'a pris, en général, le théâtre de la guerre, qui comprend souvent des terrains éloignés les uns des autres et d'un accès difficile.

On a objecté que le soldat se sert souvent des mouchoirs triangulaires qu'il porte pour autre chose que pour panser ses blessures, mais ce n'est.là qu'une affaire de surveillance et de discipline, qui ne touche pas au fond de la question.

On a observé aussi, et avec raison, qu'un premier pansement ne peut être appliqué an milieu de la mêlée du combat, et que la première chose à faire est d'éloigner les blessés pour les protéger contre de nouvelles atteintes; mais, une fois ce transport effectué, le premier pansement dont nous parlons reprend toute son importance et son utilité.

L'expérience en a été renouvelée en pariculier en 1882 en Egypte, à la bataille de Tell-El-Kébir, où le premier ransement fut appliqué avec succès à de nombreux blessés, de la manière que nous avons indiquée.

Il est cerlain, d'autre part, qu'il ne fanl pas surcharger le soldat en campagne d'un poids quelconque, ne füt-il que de deux ou trois onces, si on peut l'éviter. Il est également vrai qu'il ne faut pas augmenter les obligations de l'Etat, qui deviennent notables quand elles sont multipliées par le nombre des hommes. Mais les avantages du premier pansement individuel l'emportent, après tout, sur ses inconvénients el doivent le faire maintenir.

Une autre précaution, qu'il est indispensable de prendre, c'est de fixer règlementairement la place où devront être portés les articles de pansement, place qui doit être facilement accessible et connue de tous. Il faut qu'elle soit dans l'uniforme et non dans le havre-sac qu la valise. 
Voici, d'après les expériences de l'auteur, les conditions que ce petit matériel doit remplir :

$1^{0}$ Avoir des propriétés antiseptiques;

$2^{\circ}$ Pouvoir se conserver sans altération pendant trois ou quatre ans;

$3^{\circ}$ Etre d'une application facile et simple et pouvoir rester un ou deux jours en place sur la blessure;

4. Etre applicable à des plaies de différentes formes ;

$5^{\circ}$ Etre d'un prix modéré;

$6^{\circ}$ Etre protégé contre l'action délétère du dehors et contre l'évaporation;

$7^{\circ}$ Le paquet doit contenir deux compresses, pour les plaies d'entrée et de sortie, une attache ot un mouchoir triangulaire;

$8^{\circ}$ Le tout doit avoir une grandeur et une forme commodes pour trouver place dans l'uniforme, et ne pas répandre d'odeur désagréable.

$9^{n}$ Le contenu du paquet sera indiqué à l'extérieur.

L'auteur entre encore dans quelques détails sur la dimension des objets à introduire dans le paquet de pansement et sur lèurs propriétés désinfectantes.

A la fin du mémoire, nous trouvons la mention d'une série de prescriptions données par le professeur Esmarch, de Kiel, quant à l'utilité et au mode de composition d'un bon paquet de pansement à faire porter au soldat.

Nous terminons ici notre compte rendu de l'opuscule de Sir Thomas Longmore, mais nous ne le ferons pas sans avoir remercié l'auteur des informations et des expériences qu'il nous communique sur un sujet d'une grande importance pratique. La question est, en outre, de uature à intéresser l'œuvre de la Croix-Ronge, qui doit servir de complément à l'administration médicale officielle. Elle devra se mettre en mesure d'avoir des assortiments, pour le premier pansement, pareils à ceux que le règlement impose à chaque soldat, afin de pouvoir en fournir aux hommes qui auraient perdu ou détérioré le leur. Puis, les volontaires de la Croix-Rouge aideront les hlessés à s'en servir, el à le compléter au besoin par quelques procédés improvisés.

Nous ne voulons pas rentrer ici dans le débat en ajoutant notre opinion personnelle à celle bien plus compétente du professeur tle Netley. Nous dirons seulernent que nos propres expériences, dans 


\section{1}

plusieurs campagnes, nous ont prouvé que les objets de pansement portés par le soldat sont le complément indispensable des dépôts, même les mieux organisés, d'une armée ; c'est ainsi que, soit en 1866, en Italie, soit en 1870, en France, faute de celte ressource, nous avons dù souvent recourir à des improvisations instanta. nées.

Nous rappelons enfin que le soldat doit être initié, par quelques exercices sur lui-même et sur d'autres, a l'emploi qu'il sera appelé de faire des objets qu'il porte.

Il a paru, sur le sujet des premiers secours à se donner à soimême en cas de blessure, une petite brochure populaire illustrée, intitulée: "Von der Selbsthülfe bei Verwundungen im Kriege, von Stabsarzt Dr Diemer (Leipzig 1886). D

$D^{r}$ APpia.

\section{HESSE}

CORPS DE BRANCARDIERS DES ÉCOLES ${ }^{1}$

A l'instigation de la direction de la Société de la Croix-Ronge du grand-duché de Hesse, le ministre de l'intérieur et de la justice de cet Etat avait adressé, le 11 juillet 1888, aux directeurs des gymnases et des écoles réales, une circulaire, les invitant à provoquer, dans les établissements soumis à leur direction, la formation de corps de brancardiers volontaires. Il nous paraît intéressant, avant de parler des résultats obtenus à la suite de cette circulaire, de dire quelques mots des instructions qu'elle contenait.

Le ròle des brancardiers consiste, on le sait, non seulement à transporter les blessés et les malades, mais encore à les panser, à les coucher et à les réconforter. Cela suppose une certaine connaissance du corps humain et un enseignement théorique accompagné d'exercices pratiques. En temps de guerre, on aura besoin de brancardiers, soit auprès des armées en campagne, soit dans le pays pour le service des gares et des hôpitaux. Le recrutement du

1 Extrait du liriegerheil, janvier 1890. 